Revista de Comunicación y Salud, 2022, Vol. 12, 61-81

Editado por Cátedra de Comunicación y Salud

ISSN: 2173-1675

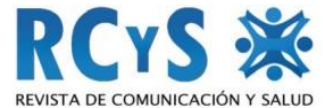

Enviado 27/05/2021

Aprobado 10/11/2021

Publicado 11/01/2022

\title{
RELEVANCIA DE LOS SISTEMAS PERSONALES DE SALUD DURANTE LA PANDEMIA DE COVID-19 EN MÉXICO
}

\section{Personal Health Records' relevance during Covid-19 pandemic in México}

Gustavo Emilio Mendoza Olguín: Benemérita Universidad Autónoma de Puebla. México.

lyondb@hotmail.com

Iván Agustín Mendoza Olguín: Instituto Mexicano del Seguro Social. México. docmoia@hotmail.com

María de la Concepción Pérez de Celis Herrero: Benemérita Universidad Autónoma de Puebla. México.

mcpcelish@gmail.com

María Josefa Somodevilla García: Benemérita Universidad Autónoma de Puebla.

México.

mariajsomodevilla@gmail.com

Financiación. Este trabajo fue financiado por el programa de Becas Nacionales de Maestría del Consejo Nacional de Ciencia y Tecnología (Conacyt) y la participación privada de profesionales de la salud.

\section{Resumen}

La educación en materia de prevención de salud representa un reto en el que México, debido a su diversidad social, cultural, geográfica, económica y política; tiene una deuda histórica. Si bien es cierto que esta situación debe enfrentarse desde varios flancos: educación, economía, condiciones de trabajo, disponibilidad de los servicios y profesionales de salud - entre muchos otros - es indudable que la tecnología debe jugar un papel importante en todos. La experiencia de otros países de establecer expedientes clínicos electrónicos universales permite comprobar que conocer el estado de salud de su población permite a los gobiernos tomar decisiones que impacten de manera positiva en la población. El objetivo de este estudio es evaluar la reacción de los actores sociales (pacientes y médicos) a un sistema personal de salud. Con este fin se desarrolló un Sistema Personal de Salud basado en Android y compatible con el estándar HL7 v3 y se comparó contra su equivalente en papel. Se agrega el contexto de la pandemia causada por el Covid-19 debido a que durante la fase de pruebas el 


\section{RELEVANCIA DE LOS SISTEMAS PERSONALES DE SALUD DURANTE LA PANDEMIA DE COVID-19 EN MÉXICO}

país comenzó con la primera ola de contagios; lo que afectó de cierta forma el número de pruebas, pero a la vez demostró la importancia de estas herramientas en situaciones críticas. Los resultados permiten hacer inferencias importantes sobre las características que debe tener este tipo de herramientas para funcionar en el mercado mexicano.

Palabras clave: Sistema personal de salud, Expediente clínico, HL7, covid-19

Education about health prevention represents a challenge in which Mexico, due to its social, cultural, geographical, economical and political diversity, is historically behind. Although it is true that this situation must be confronted from many sides: education, culture, economy, work conditions, health services and professionals availability among others - it is unquestionable that technology must play an important role in each one. The experience from other countries that already use electronic clinical records for its population can prove that they meet the health status of their people and allow the government to make decisions with a positive impact for them. The main objective of this work is to evaluate the reactions of the social actors (doctors and patients) in regards to Personal Health Records. In order to do this, an android-based HL7v3compatible PHR was developed and compared against its paper equivalent. The COVID-19 pandemic context was added because during the test phase, the country experienced the first wave of contagion. This affects the number of tests but also proves the importance of these tools in critical situations. The results allow us to make inferences about the characteristics that these kinds of tools must have in order to be functional in the Mexican market.

Keywords: Personal health record, clinical record, HL7, covid-19.

\section{INTRODUCCIÓN}

Un Registro Personal de Salud (también conocido como PHR por Personal Health Record) es un conjunto de herramientas basadas en tecnologías de la información que permiten a las personas concentrar y administrar su información de salud durante el transcurso de su vida, con la posibilidad de compartir fragmentos de esta información si así lo requiere. (Kaelber et al., 2008). El concepto aparentemente simple ha sido implementado sin embargo con cierta dificultad en los diferentes países donde se tienen herramientas de este tipo (Estados Unidos, Alemania, Australia, etc); ya que la legislación, requerimientos (técnicos, de privacidad y seguridad, confiabilidad y de adherencia de los usuarios) y funcionalidades que deben considerarse representan retos que deben solventarse en diferentes niveles. En el contexto mexicano el mayor acercamiento hacia una herramienta de este tipo, aunque no puede llamarse completamente un PHR, se dio en 2018 cuando la Secretaría de Salud publicó RadarCISalud (Secretaría de Salud, 2018), como una herramienta de búsqueda rápida de servicios de salud basados en la localización y que incluye entre sus características un expediente clínico básico. 


\section{RELEVANCIA DE LOS SISTEMAS PERSONALES DE SALUD DURANTE LA PANDEMIA DE COVID-19 EN MÉXICO}

\subsection{Descripción funcional de un PHR}

De acuerdo al concepto, un PHR debe ofrecer como mínimo: a) un registro temporal (bitácora/journal) donde el usuario anote, de forma dinámica y con la periodicidad que desee, los eventos de salud que considere importantes; abarcando todas las áreas de la salud; b) un registro permanente (expediente) de la información de salud que incluya tanto los datos variantes (talla, peso, presión arterial, etc.) como invariantes en el tiempo (grupo sanguíneo, padecimientos congénitos, etc.); obtenidos automática o manualmente. Y c) La capacidad de compartir la cantidad de información deseada con los actores seleccionados por el usuario en diferentes formatos (texto, imagen, registros electrónicos, etc.)

La AHIMA (AHIMA e-HIM Personal Health Record Work Group., 2005) estableció las características que debe cumplir un PHR y las agrupa en 5 aspectos: funcionalidad, formato y contenido, privacidad y control de acceso, mantenimiento y seguridad e interoperabilidad. En el caso de que el PHR esté interconectado a un Expediente Médico Electrónico (EMR de Electronic Medical Record), este debe poder manejar como mínimo la información que solicita el EMR, pero no existe una cota superior. En el contexto de México, la falta de un Expediente Clínico Electrónico universal implica que no existe tampoco una cota mínima sobre la información para los PHR destinados al uso general. Sin embargo Leal y su equipo (Leal et al., 2011) apuntan, con respecto el Expediente Clínico Electrónico, que la información debe cumplir con los datos establecidos en la Norma Oficial Mexicana del Expediente Clínico.

\subsection{La evolución del expediente clínico nacional.}

La Constitución Política de los Estados Unidos Mexicanos en su artículo cuarto garantiza el derecho a la salud y la igualdad ante la ley de todos los mexicanos. (Constitución Política de los Estados Unidos Mexicanos, 1917). En consecuencia, es obligación del Estado Mexicano garantizar el funcionamiento del Sistema de Salud Nacional, la figura 1 muestra su conformación actual. 


\section{RELEVANCIA DE LOS SISTEMAS PERSONALES DE SALUD DURANTE LA PANDEMIA DE COVID-19 EN MÉXICO}

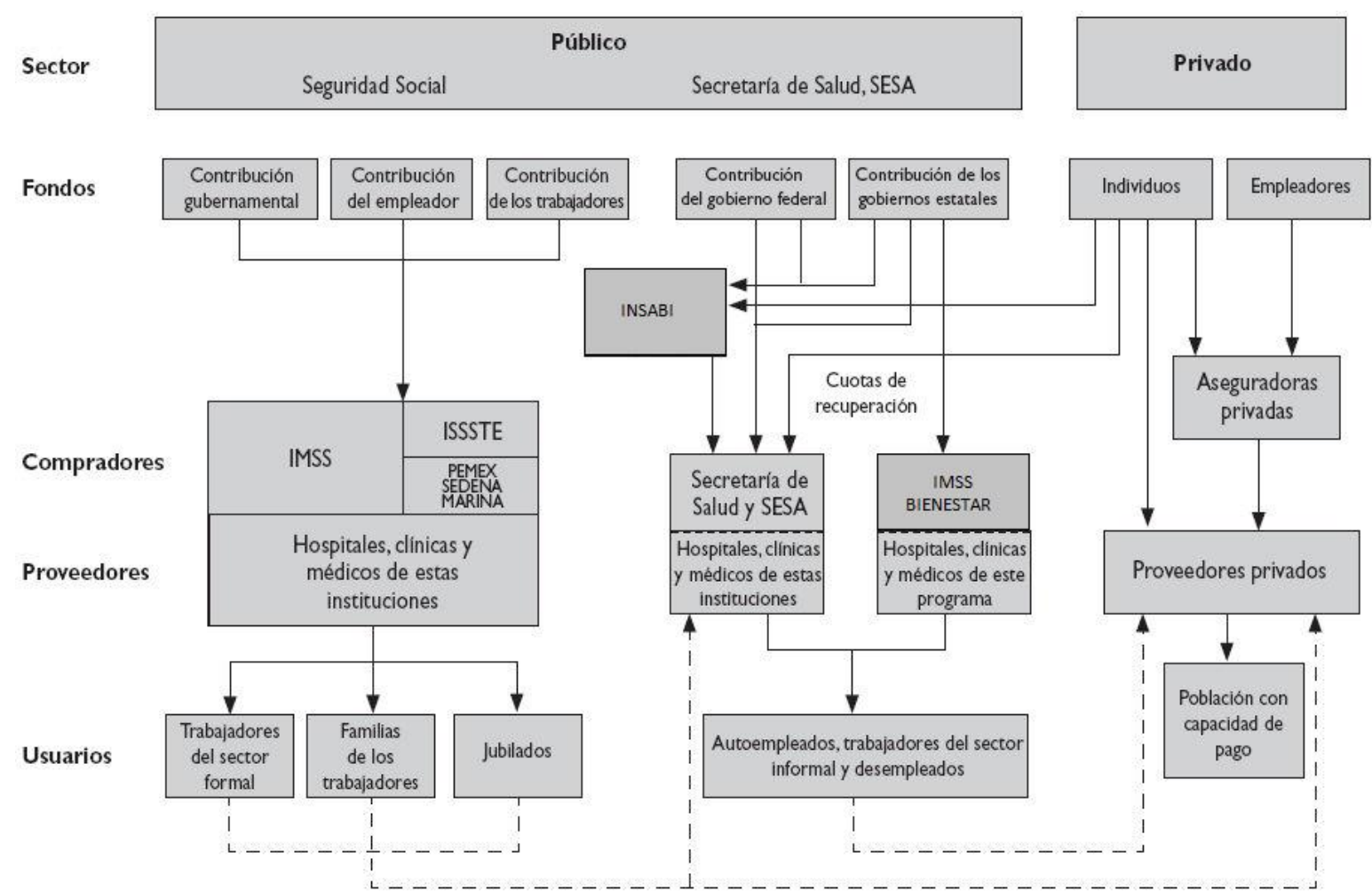

Figura 1. El sistema de salud nacional.

Fuente: Actualización propia basada en (Dantés et al., 2011)

Las instituciones de Seguridad Social que ofrecen servicios a los trabajadores que forman parte del sector formal de la economía, junto con las fuerzas armadas y sus familias, el sector petrolero y los burócratas conforman el sector público. (Dantés et al., 2011). Adicionalmente, hay otros organismos de este mismo sector enfocados a ciudadanos que no tienen seguridad social, como la Secretaría de Salud (SSA), el INSABI, los Servicios Estatales de Salud (SESA) y el programa IMSS-BIENESTAR (antes IMSS-Oportunidades) que está dirigido a comunidades rurales e indígenas que no cuentan con seguridad social (Instituto Mexicano del Seguro Social, 2019). El financiamiento de este sector proviene de los gobiernos federal y estatales, aunque pueden percibir cuotas de recuperación por los servicios prestados.

La población con mayores ingresos económicos utiliza regularmente el sector privado. Este se encuentra conformado por clínicas, hospitales, laboratorios y consultorios particulares que se financian principalmente por el pago de los pacientes por evento e ingresos externos que pueden provenir de primas de los seguros médicos privados y la subcontratación de servicios entre sectores. En estos servicios se incluyen también los profesionales médicos independientes.

Actualmente la elección de qué sectores para la atención en salud utilizar, depende totalmente del paciente: la población con menores ingresos económicos se atiende en 


\section{RELEVANCIA DE LOS SISTEMAS PERSONALES DE SALUD DURANTE LA PANDEMIA DE COVID-19 EN MÉXICO}

instituciones públicas, lo que se traduce en saturación y tiempos de espera largos. Los grupos de mayor poder adquisitivo ven al sector público como la opción para tratamientos de urgencia o enfermedades degenerativas de largo plazo, pero para eventos de salud en general se decantan por utilizar el sector privado. Finalmente, la población con mayores ingresos considera deficiente a las instituciones públicas y optan por la atención privada en todos los casos. La contingencia causada por el virus SARS-COV2 ha sido útil para evidenciar los beneficios y las áreas de oportunidad de ambos sectores; pero es evidente que las enfermedades degenerativas que conforman las primeras causas de muerte de los mexicanos los colocan en una situación de vulnerabilidad comparado con otros países (Navarro, 2020).

Las instituciones del sector privado han sido punta de lanza en el uso y la implementación de HIS (Health Information Systems) internos o bien adquiridos a empresas especializadas con HIS genéricos adaptables. Ante el surgimiento de sistemas con características variadas en cuanto a formato y contenido, la Secretaría de Salud fue instruida a establecer recomendaciones que permitieran a las instituciones escoger de mejor manera los sistemas a implementar mediante las características que se apegan a la Guía de Intercambio de Información de Salud (GIIS) (Dirección General de Información en Salud, 2011). El marco regulatorio de estas recomendaciones abarca tanto el contenido, como el formato y la seguridad de la información y está asentado en la legislación vigente y varias normas oficiales establecidas para estandarizar los procedimientos relacionados con la salud de los mexicanos, cuyo carácter de obligatoriedad es nacional. Entre estas normas destacan la que establece los objetivos funcionales y funcionalidades que deben observar los productos de Sistemas de Expediente Clínico Electrónico para garantizar la interoperabilidad, procesamiento, interpretación, confidencialidad, seguridad, uso de estándares y catálogos de la información de los registros electrónicos en Salud (Secretaría de Salud, 2012); En esta se establece a la Secretaría de Salud, basada en las disposiciones jurídicas aplicables, como el organismo rector de la normatividad a la que deben ajustarse las Unidades que forman el Sistema Nacional de Salud en lo referente a los Sistemas de Expediente Clínico Electrónico. Dicho sistema debe garantizar la integridad y confiabilidad de la información clínica, la confidencialidad e identidad de los pacientes, así como establecer los mecanismos de seguridad pertinentes y adecuados con la finalidad de prevenir el uso ilícito o ilegítimo que pueda resolver la esfera jurídica del titular de la información; además de cumplir obligatoriamente con los elementos que se mencionan tanto como requeridos y sugeridos. El manejo del ECE deberá realizarse con discreción y confidencialidad y la información contenida solo puede ser dada a conocer al paciente o a quien tenga facultad legal para decidir por él y, en su caso, a terceros mediante una orden judicial o administrativa competente. Aunque esta norma representa, indudablemente, un avance que favorece el establecimiento de un expediente clínico electrónico (ECE) universal, queda claro que el ECE no es aún propiedad de paciente y tiene acceso a éste sin restricciones. 


\section{RELEVANCIA DE LOS SISTEMAS PERSONALES DE SALUD DURANTE LA PANDEMIA DE COVID-19 EN MÉXICO}

La penetración de las tecnologías de la información y comunicación en todos los ámbitos del trabajo diario se ha visto reflejado también en el manejo de la información de salud, Actualmente, pueden encontrarse expedientes clínicos en papel sólo en las instituciones públicas y en las áreas donde es evidente su ventaja sobre los medios electrónicos. Sin embargo, los hospitales privados han mostrado ir adelantados en cuanto a la implementación y manejo de Sistemas de Información de Salud (HIS, Health Information Systems), lo que obligó a las autoridades a establecer recomendaciones y normativas que regularan contenido, formato y seguridad de la información de salud bajo un marco jurídico de obligatoriedad nacional. Las mas importantes son la Guía de intercambio de información de Salud (GIIS) (Dirección General de Información en Salud, 2011), la Norma Oficial Mexicana NOM-024-SSA3-2012 (Secretaría de Salud, 2012) y la Guía de Intercambio de Información para el intercambio de resúmenes clínicos en formato electrónico (Dirección General de Información en Salud, 2016), la cual establece que el estándar de intercambio de información será Health Level 7 version 3 (HL7 v3) y estandariza la interoperabilidad de los diferentes SIRES (Sistemas de Información de Registro Electrónico para la Salud). Esta guía también establece la plantilla final del documento con los valores asociados para México; lo que permite inferir otros datos requeridos pues el resumen clínico puede entenderse como un extracto del expediente clínico.

\subsection{El estándar Health Level 7.}

De acuerdo con la HL7 International, Health Level $7(H L 7)$ es "un estándar internacional para el intercambio, integración, intercambio y recuperación de información electrónica de salud, incluyendo práctica clínica, y la administración, entrega y evaluación de servicios de salud" (HL7 International, 2019). Fue propuesto en 1987 y actualmente es utilizado en 50 países. Ofrece herramientas y frameworks que facilitan su uso e integración en sistemas de información de forma gratuita y de libre uso.

La versión 3 del estándar puede entenderse como un intercambio de elementos llamados mensajes, los cuales son contenedores de subsecciones que dependen del tipo de mensaje y de los actores involucrados. La especificación estructurada de la información dentro de un dominio específico de interés recibe el nombre de modelo de información, el cual expresa las clases de información requerida y las propiedades de esas clases, incluyendo atributos, relaciones, restricciones y estados. (HL7 Organization, 2019, p. 7)

Según la documentación del estándar, un modelo de información está compuesto por las clases, sus atributos y relaciones, los tipos de datos de los atributos y el dominio del vocabulario y los modelos de transición de estados requeridos. Los modelos de información que ofrece HL7v3 pueden clasificarse en tres tipos que se diferencian en la información contenida, el alcance y uso de la misma. Estos modelos son: 


\section{RELEVANCIA DE LOS SISTEMAS PERSONALES DE SALUD DURANTE LA PANDEMIA DE COVID-19 EN MÉXICO}

- RIM (Reference Information Model): Es un modelo compartido, coherente y abstracto que funciona como el origen de todos los datos que conforman los diferentes mensajes utilizados. Representa de forma abstracta la riqueza de contenidos de los tópicos que pueden compartirse a través de un HIS. La armonización del modelo se realiza por los comités técnicos y los grupos involucrados.

- DMIM (Domain Message Information Model): Contiene copias de las clases, atributos y relaciones que se utilizarán en un determinado dominio (área de la salud), las cuales se obtienen del RIM. Funciona cómo el modelo inicial de los RMIMS que se utilizarán dentro de un dominio específico.

- RMIMS (Refined Message Information Model): representa un subconjunto de un DMIM utilizado para expresar el contenido, anotaciones y refinamientos específicos de un mensaje o un conjunto de mensajes.

El proceso de diseño de un PHR apegado al estándar no requiere utilizar todo el RIM de forma obligatoria, sino solo delimitar el dominio de trabajo mediante un DMIM y, basándose en éste, construir el RMIM de acuerdo al intercambio de mensajes que se necesiten.

En esta investigación se evalúa el grado de aceptación de un PHR entre los actores involucrados: pacientes y médicos. Por el lado de los pacientes el PHR evaluado ofrece la sustitución de los registros en papel, agregando elementos como seguridad, privacidad y facilidad para compartir la información almacenada con el profesional de salud de su confianza. Por otro lado, se ofrece a los profesionales de salud una herramienta que le permite conocer todo el historial completo del paciente (si el PHR es alimentado de forma adecuada). Adicionalmente, se pretende identificar cuáles pueden ser las causas que pueden interferir con el éxito de un PHR en el contexto nacional. La metodología utilizada con ambos actores se presenta en la siguiente sección.

\section{METODOLOGÍA}

Previo al desarrollo y prueba del PHR motivo de esta investigación se realizaron dos estudios de tipo descriptivo-exploratorio. Para el primero se utilizó como instrumento una encuesta realizada por internet cuyas categorías fueron los datos sociodemográficos, hábitos de salud y la disposición a utilizar un PHR de personas mayores de 18 años. Para el segundo, el instrumento utilizado fueron entrevistas a médicos de diferentes especialidades. Las categorías consideradas en este instrumento fueron la valoración personal sobre los PHR y las características que desde su especialidad considerarían imprescindibles para que estas herramientas fueran consideradas funcionales.

Es importante mencionar que, debido a la naturaleza de las respuestas de ambos estudios, los resultados son dependientes de la técnica de muestreo utilizada. Para el primer estudio se utilizó un muestre probabilístico de tipo aleatorio simple cuya única 


\section{RELEVANCIA DE LOS SISTEMAS PERSONALES DE SALUD DURANTE LA PANDEMIA DE COVID-19 EN MÉXICO}

restricción fue la mayoría de edad. Para el segundo se utilizó un muestreo no probabilístico de tipo a conveniencia de médicos con diferentes especialidades que aceptaron la entrevista.

El primer estudio mostro que el $56.3 \%$ de los encuestados habían visitado al médico en los últimos seis meses. Sin embargo, el $77.9 \%$ de los encuestados contestaron que sus visitas al médico eran cada que tenían una molestia, y sólo el $15.7 \%$ tenían visitas programadas de prevención, los resultados fueron similares a la pregunta sobre el sector utilizado. Esta encuesta también permitió definir que la mayoría de los usuarios, el $86.4 \%$ de los encuestados, se mostró interesado en utilizar un PHR en su celular.

Del segundo estudio se obtuvo que el $82 \%$ de los encuestados utilizaría la aplicación si ésta le permitiera generar la documentación requerida por las autoridades supervisoras, aunque también un gran porcentaje, el $70 \%$, mencionó preocupación por el grado de relevancia de los datos que los pacientes entregaran. También fue notoria la gran variedad de información requerida entre las diferentes las ramas de la medicina. Esto motivó a que fuera necesario definir los límites de la app en su primera versión. Con esto se optó por implementar dos componentes: La bitácora médica personal y el registro médico personal. La tabla 1 muestra la finalidad de cada componente.

Tabla 1. Finalidad de los módulos de la aplicación

\begin{tabular}{|c|c|}
\hline Bitácora médica personal & Registro médico personal \\
\hline $\begin{array}{l}\text { Estadísticas basadas en la información } \\
\text { almacenada por el usuario; por ejemplo: } \\
\text { gráficos de comportamiento de presión } \\
\text { arterial, nivel de azúcar en sangre, o } \\
\text { análisis de padecimientos reportados. }\end{array}$ & $\begin{array}{l}\text { Información médica de emergencia y } \\
\text { contacto multiformato. }\end{array}$ \\
\hline $\begin{array}{l}\text { Mediante el uso de bases de } \\
\text { conocimiento validadas, } \\
\text { recomendaciones ofrecer } \\
\text { conocimiento tras el anadas } \\
\text { información almacenada. }\end{array}$ & $\begin{array}{l}\text { Historial clínico: intervenciones } \\
\text { quirúrgicas, citas médicas, tratamientos } \\
\text { completados y en curso. }\end{array}$ \\
\hline & $\begin{array}{l}\text { Historial de inmunizaciones de acuerdo a } \\
\text { edad del usuario. }\end{array}$ \\
\hline
\end{tabular}

Fuente: Elaboración propia.

Para la fase de pruebas, se propuso trabajar con diferentes especialistas para que propusieran el usar la aplicación a un grupo de pacientes seleccionados por ellos. Adicionalmente se ofreció utilizar la aplicación a personas desconocidas por los médicos, para probar si la información recibida permitía elaborar un diagnóstico de salud. Por considerarse información privilegiada, todos los involucrados debían firmar un consentimiento para poder trabajar con su información y que ésta fuera revisada por un médico que no fuera el de su confianza. Esta fase consta de dos etapas: la primera 


\section{RELEVANCIA DE LOS SISTEMAS PERSONALES DE SALUD DURANTE LA PANDEMIA DE COVID-19 EN MÉXICO}

de seguimiento en papel y la segunda que incluye un seguimiento digital mediante una app.

Un modelo esquematizado de la app se encuentra en la figura 2.

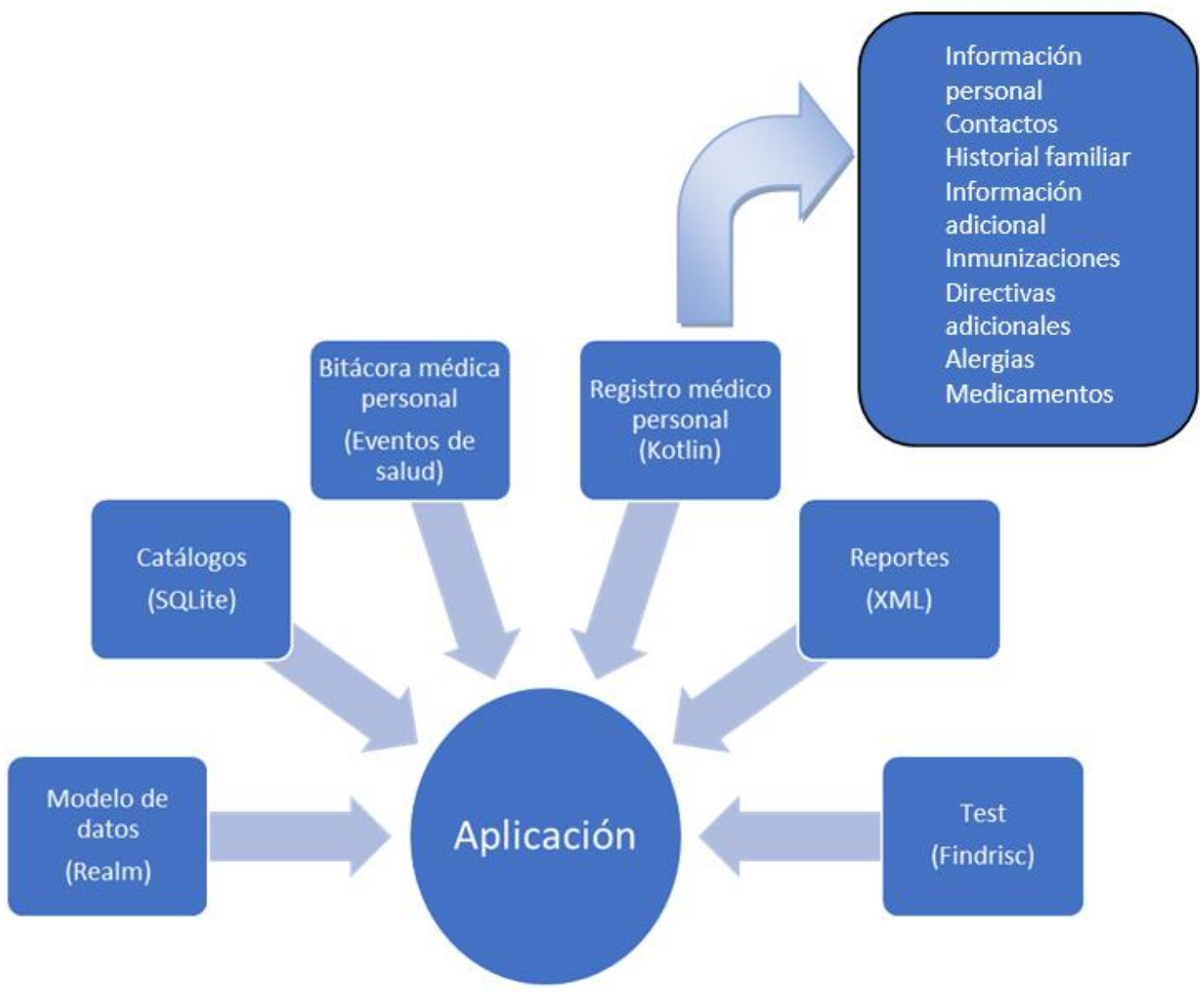

Figura 2. Esquema del PHR.

Fuente: Elaboración propia.

\subsection{Arquitectura de base de datos.}

Para el almacenamiento de la información se comparó un Sistema Gestor de Bases de Datos (SGBD) relacional y un SGBD NoSQL. Analizando sus ventajas y desventajas, se obtuvo que un SGBD relacional en sencillo en su implementación sobre Android utilizando las opciones existentes (SQLite, Room). SQLlite ofrece un kit de desarrollo de software ( $S D K$, Software Development Kit) listo para trabajar con varios entornos de desarrollo; lo que simplifica la recuperación de información. La principal desventaja de este modelo es que, por efecto de la dispersión, las tablas aumentarán 


\section{RELEVANCIA DE LOS SISTEMAS PERSONALES DE SALUD DURANTE LA PANDEMIA DE COVID-19 EN MÉXICO}

los requerimientos de almacenamiento con el tiempo sin que esto represente un incremento en la información relevante.

Por su parte los modelos de SGBD noSQL solucionan el requerimiento de almacenamiento debido a que sus estructuras sólo pueden aumentan su tamaño conforme se agrega información. Analizando entre diferentes herramientas, se decantó por Realm (Realm, 2019). Esta es una implementación open source NoSQL que utiliza un enfoque de elementos par:valor organizados en documentos, similares a los utilizados por MongoDB; es orientado a objetos e incluye un lenguaje de consulta nativo. Adicionalmente, ofrece encriptación AES-256 que proporciona seguridad a la información y soporta transacciones síncronas y asíncronas.

Dentro de las consideraciones de implementación, el tipo de relaciones que pueden establecerse entre objetos puede llevar a duplicidad de valores si es que se utiliza el modo de referencia incrustada, en este modelo, un objeto relacionado se encuentra dentro de otro objeto que lo contiene; esto se puede resolver si se utiliza una relación por referencia, lo que permite la reutilización de los objetos; sin embargo, con este modelo de referencias pueden existir problemas de rendimiento pues a consulta puede requerir múltiples transacciones en orden de obtener toda la información de los objetos relacionados. Además, debe recordarse la importancia de cerrar explícitamente las transacciones a la base de datos pues estas no se cierran automáticamente, lo que puede ocasionar incrementos de uso de memoria.

Por la arquitectura de Room, es necesario crear clases que sean directamente objetos Realm. Los datos que pueden contener incluyen valores, documentos, arreglos y arreglos de arreglos. Se determinó que cada entrada a la bitácora fuera un objeto separado que solo será incluido en el expediente si el usuario lo determina; lo que ayuda a cumplir con el requerimiento del control de información. La limitación física de que una cadena de caracteres no pueda exceder los $16 \mathrm{Mb}$ de tamaño obliga a que cierto tipo de formatos de uso médico como DICOMM o las imágenes deban ser manejados como elementos externos a la base de datos.

\subsection{Integración del estándar}

El objetivo final de la app es la construcción del expediente médico personal. De acuerdo con el estándar HL7v3, el expediente fue considerado como un mensaje compuesto a su vez por otros submensajes de diferente tipo, operaciones y estructura, pero que utilizan XML como lenguaje común. Se utilizó la implementación propuesta por HL7 para el intercambio de información de PHR a PHR (HL7 International, 2011) realizando las adecuaciones requeridas por la GIIS-A001-01-05 y con la NOM-024SSA3-2012. La estructura del documento se muestra en la figura 3. 


\section{RELEVANCIA DE LOS SISTEMAS PERSONALES DE SALUD DURANTE LA PANDEMIA DE COVID-19 EN MÉXICO}

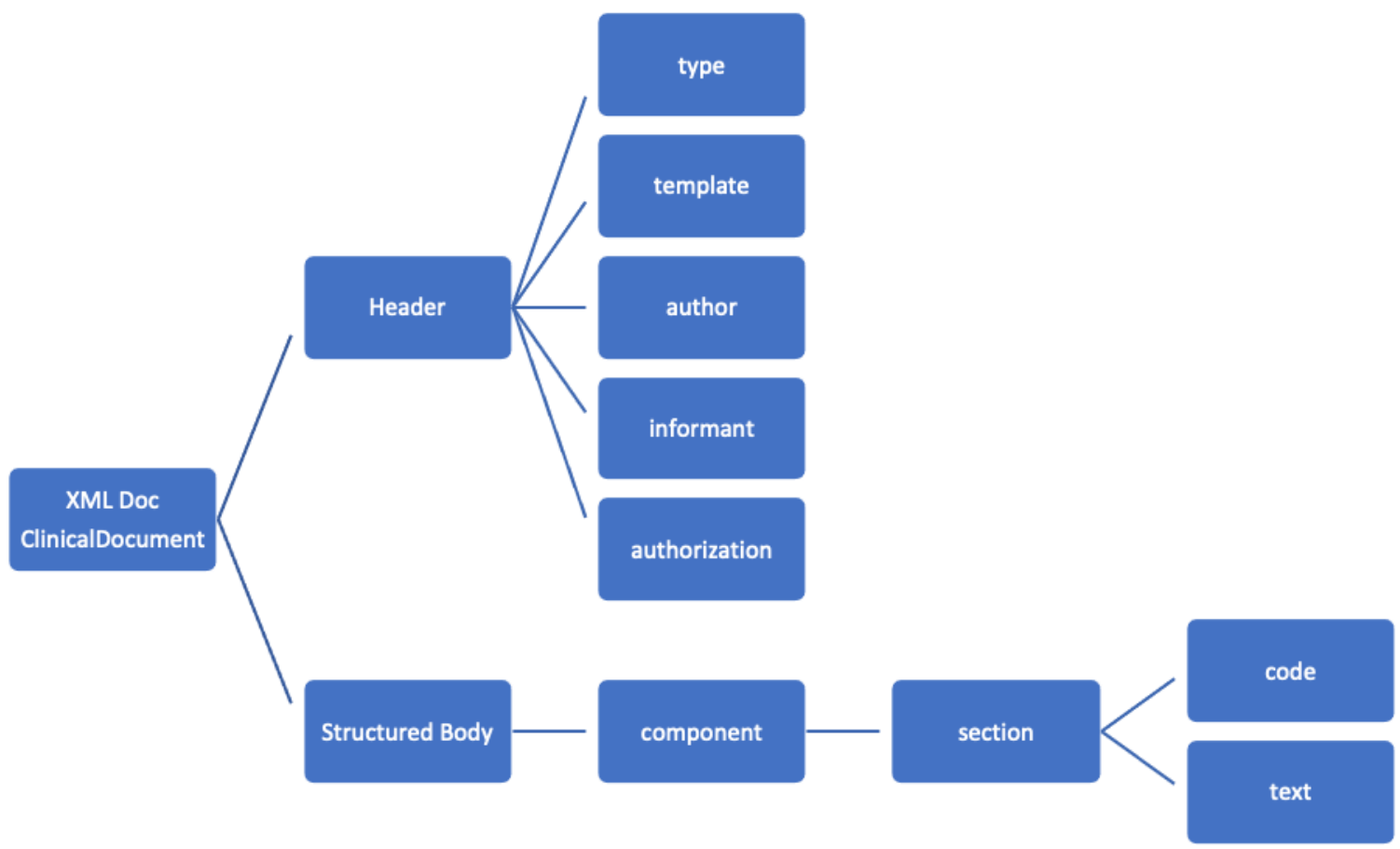

Figura 3. Estructura del documento XML para el intercambio de información entre PHR's de acuerdo al estándar HL7-CDA2

Fuente: Elaboración propia basada en (HL7 International, 2011)

Basado en lo mencionado anteriormente, un documento clínico contiene dos elementos: una cabecera o header y un cuerpo estructurado (structuredBody). Las plantillas XML que definen como se organizan las diferentes secciones están estructuradas bajo el estándar CDA2 y se distinguen mediante un templateld. La legibilidad de la información por actores humanos y no humanos es un requerimiento esencial. La la tabla 2 se presentan las secciones requeridas, los templatelDs y el contenido de los mismos.

Tabla 2. Secciones consideradas para el documento XML

\begin{tabular}{|l|c|l|}
\hline \multicolumn{1}{|c|}{ Sección } & \multicolumn{1}{|c|}{ templateld } & \multicolumn{1}{c|}{ Contenido } \\
\hline $\begin{array}{l}\text { Advanced } \\
\text { directives } \\
\text { section }\end{array}$ & 2.16 .840 .1 .113883 .10 .20 .1 .1 & $\begin{array}{l}\text { Indicaciones del paciente y sus } \\
\text { referencias. }\end{array}$ \\
\hline $\begin{array}{l}\text { Alerts and } \\
\text { allergies } \\
\text { section }\end{array}$ & 2.16 .840 .1 .113883 .10 .20 .1 .2 & Alergias conocidas. \\
\hline $\begin{array}{l}\text { Encounters } \\
\text { section }\end{array}$ & 2.16 .840 .1 .113883 .10 .20 .1 .3 & Visitas médicas. \\
\hline $\begin{array}{l}\text { Family history } \\
\text { section }\end{array}$ & 2.16 .84 .1 .113883 .10 .20 .1 .4 & $\begin{array}{l}\text { Historia familiar contenida en } \\
\text { una estructura organizer. }\end{array}$ \\
\hline
\end{tabular}




\section{RELEVANCIA DE LOS SISTEMAS PERSONALES DE SALUD DURANTE LA PANDEMIA DE COVID-19 EN MÉXICO}

\begin{tabular}{|l|c|l|}
\hline $\begin{array}{l}\text { Immunization } \\
\text { section }\end{array}$ & 2.16 .840 .1 .113883 .10 .20 .1 .6 & Historial de vacunación. \\
\hline $\begin{array}{l}\text { Medication } \\
\text { section }\end{array}$ & 2.16 .840 .1 .113883 .10 .20 .1 .8 & $\begin{array}{l}\text { Medicamentos consumidos. } \\
\text { Automedicaciones } \\
\text { homeopatías. }\end{array}$ \\
\hline $\begin{array}{l}\text { Plan of care } \\
\text { section }\end{array}$ & 2.16 .840 .1 .113883 .10 .20 .1 .10 & $\begin{array}{l}\text { Encuentros, procedimientos, } \\
\text { tratamientos o indicaciones } \\
\text { actuales. }\end{array}$ \\
\hline $\begin{array}{l}\text { Problems } \\
\text { section }\end{array}$ & 2.16 .840 .1 .113883 .10 .20 .1 .1 & $\begin{array}{l}\text { Problemas que se presentaron } \\
\text { al crear el documento. }\end{array}$ \\
\hline $\begin{array}{l}\text { Procedures } \\
\text { section }\end{array}$ & 2.16 .840 .1 .113883 .10 .20 .1 .14 & $\begin{array}{l}\text { Hesultados de laboratorio. } \\
\text { procedimientos. }\end{array}$ \\
\hline $\begin{array}{l}\text { Results } \\
\text { section }\end{array}$ & 2.16 .840 .1 .113883 .10 .20 .8 .1 .1 & $\begin{array}{l}\text { Información personal y social y } \\
\text { hábitos de riesgo }\end{array}$ \\
\hline $\begin{array}{l}\text { Social history } \\
\text { section }\end{array}$ & 2.16 .840 .1 .113883 .10 .20 .1 .16 & Mediciones y signos vitales. \\
\hline $\begin{array}{l}\text { Vital signs } \\
\text { section }\end{array}$ & \begin{tabular}{l} 
Renes \\
\hline
\end{tabular} \\
\hline
\end{tabular}

Fuente: (HL7 International, 2011)

Fueron necesarias adaptaciones a las plantillas para que el documento XML cumpliera con la norma NOM-004-SSA3-20, de manera que, al cumplir con los requerimientos de un expediente clínico, este documento sea útil para los médicos y así promover la aceptación. Se comprobó también que los requerimientos de la norma NOM-024-SSA3-2010, que regula la interoperabilidad de los sistemas electrónicos de expediente clínico y de la GIIS-A001-01-05 fueran satisfechos, tomando en consideración que las diferencias que existen entre los expedientes clínicos y los resúmenes clínicos. Entre las adecuaciones importantes figuran la inclusión del OID (Object Identifier) para la CURP por ser un elemento obligatorio en la normativa nacional; mismo caso con la edad, que no figura en el estándar internacional y la forma de capturar los domicilios, para estos últimos se incluyeron las consideraciones de ambos estándares.

El uso del archivo de formato XSD incluido en la GIIS-A001-01-05 y marcado como recomendable también fue considerado para el despliegue del XML en navegadores compatibles. Ambos archivos, el XML y el XSD, se comparten en texto plano, sin embargo, es importante establecer algunas estrategias de seguridad para la información, las cuales se describen en la siguiente sección.

\subsection{Estrategia de protección de la información}

Por la naturaleza de la información, es importante mencionar que todos los datos solicitados forman parte de los datos protegidos por las leyes aplicables. Estas legislaciones varían entre países y en algunos casos incluso entre estados. El estándar 


\section{RELEVANCIA DE LOS SISTEMAS PERSONALES DE SALUD DURANTE LA PANDEMIA DE COVID-19 EN MÉXICO}

HL7 establece que la información contenida en la representación XML debe contener información entendible para actores humanos y no humanos, y no establece ningún elemento de cifrado para sus documentos. Para la aplicación se estableció un sistema de usuario y contraseña en un conjunto de tablas independiente de la estructura donde se encuentran almacenados los datos del usuario. La base de datos se encuentra protegida con el cifrado de 512 bits que ofrece Realm, cuya sesión se inicia únicamente ante un ingreso de credenciales correcto.

Si bien los XML no se cifran de ninguna manera, estos son enviados desde la aplicación hacia el correo electrónico asociado al profesional de salud, por lo que toda la responsabilidad de la información es de quien la genera.

\section{Resultados}

Para la obtención de los resultados se consideraron treinta personas agrupadas de la siguiente forma: diez de ellos con enfermedades crónico-degenerativas bajo la supervisión del médico tratante, por lo que ya contaban con un expediente clínico; diez de ellas que habían comentado que consideraban tener un buen estado de salud y el resto se consideraban a sí mismos como "enfermizos", pero sin seguimiento médico. A cada uno de ellos se le asignó un número para mantener la anonimidad de la información y firmaron un consentimiento para que su información fuera revisada por un médico y la intención de participar en las dos fases de la evaluación. Se utilizó un tipo de muestreo deliberado donde el criterio de selección fue que los participantes supieran leer y escribir para la primera fase y que tuvieran un teléfono celular inteligente para la segunda etapa. La primera etapa tuvo la duración de un mes durante el cual las personas registraron sus eventos de salud en un formulario diario mensual en papel.

La situación de pandemia y cuarentena causada por el virus SARS-COV2 limitaron el número de personas que participaron en la etapa de pruebas, asimismo de los profesionales de salud involucrados. Las edades de las personas seleccionadas oscilaron entre los 18 y los 55 años. La edad mínima fue considerada pues los menores de edad no pueden dar consentimiento para compartir su información de salud; la edad máxima fue considerada por el analfabetismo tecnológico que pudieran tener algunos participantes para la segunda etapa del experimento. En cuanto a los profesionales médicos, solo se obtuvo el apoyo de uno: el Dr. Iván Agustín Mendoza Olguín, con especialidad en urgencias y cédula profesional de especialidad 6249568.

En la primera fase del estudio se obtuvieron los siguientes indicadores:

- Formularios llenados en su totalidad por grupo de edad.

- Motivos para no llenarlo (mediante una pregunta abierta).

- Calidad de la información obtenida valorada por el profesional de salud. 


\section{RELEVANCIA DE LOS SISTEMAS PERSONALES DE SALUD DURANTE LA PANDEMIA DE COVID-19 EN MÉXICO}

Para su análisis, se definieron 4 grupos de edades de acuerdo al rango de valores de este dato y se clasificaron los formatos. No se consideró a qué clasificación inicial (saludable, enfermizo, degenerativo) pertenecía cada paciente. De acuerdo al gráfico 1, la mayor incidencia de formularios incompletos estuvo entre los participantes de 18 a 27 años. Ellos mencionaron como principales causas de falta de llenado la dificultad del formulario para su llenado y la falta de eventos importantes para agregar al formulario (aunque dos personas que no llenaron presentaban una condición crónicodegenerativa). Por el contrario, las personas de 48 a 55 años fueron las que mostraron un menor número de formularios incompletos. Las razones que dieron los participantes que no cumplieron fueron el olvido y el exceso de actividades diarias.

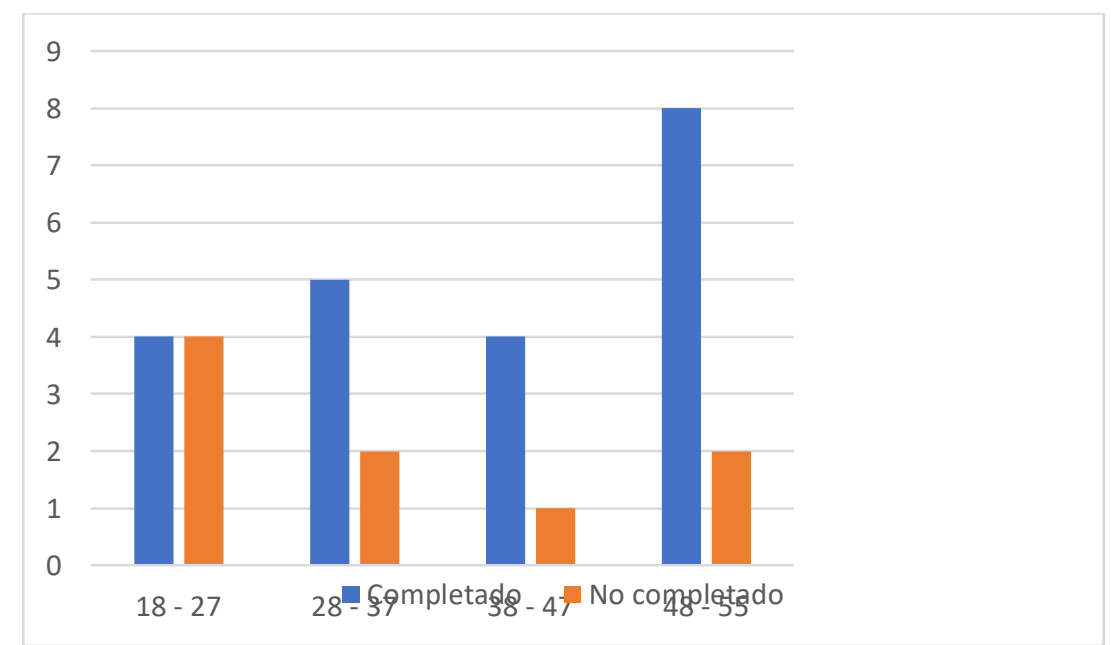

Gráfico 1 Formularios completados y no completados de la primera etapa

Los formularios completados fueron proporcionados al profesional médico, el cual al evaluarlos proporcionó retroalimentación. El profesional de salud clasificó la información recibida de acuerdo a su utilidad para elaborar un diagnóstico: Información irrelevante o incompleta, información relevante pero insuficiente, información de personas que no requieren atención médica e información de personas que requieren atención médica. Con esto se pretende evaluar de forma cualitativa la calidad de la información proporcionada por los pacientes. La evaluación recibida se presenta en la gráfica 2. 


\section{RELEVANCIA DE LOS SISTEMAS PERSONALES DE SALUD DURANTE LA PANDEMIA DE COVID-19 EN MÉXICO}

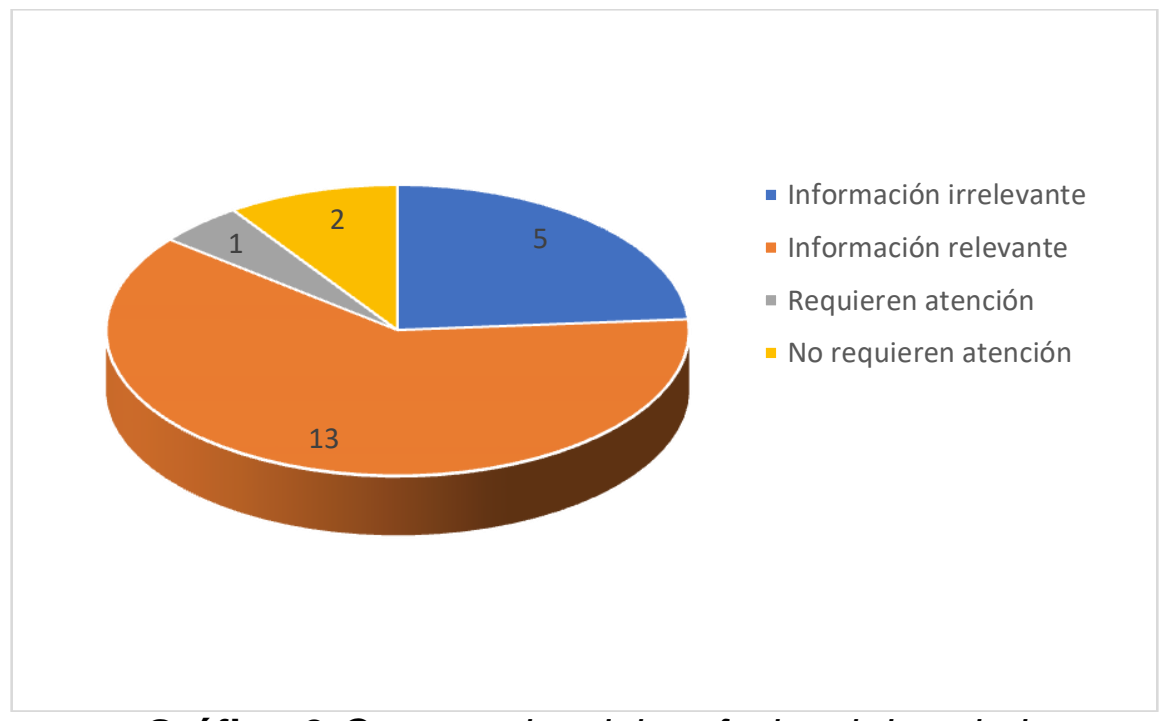

Gráfico 2 Comentarios del profesional de salud

Para obtener los resultados de la segunda etapa se diseñó una app que permitía a los usuarios registrar datos de historia familiar, factores de riesgo, alergias, anotaciones personales, encuentros de salud y mediciones. Se instaló en el teléfono celular de los voluntarios y se solicitó que la utilizaran y alimentaran durante el mismo periodo de tiempo. Al final del periodo, el XML obtenido se envió al médico para su valoración.

Los resultados obtenidos mostraron que las personas jóvenes tuvieron menos problemas en utilizar la app y registrar sus eventos, mientras que los adultos mayores tuvieron más problemas, lo cual era un resultado esperado. Sin embargo, las opiniones de estas personas fueron las más valiosas en cuanto a la usabilidad de la aplicación. Los resultados obtenidos se muestran en el gráfico 3.

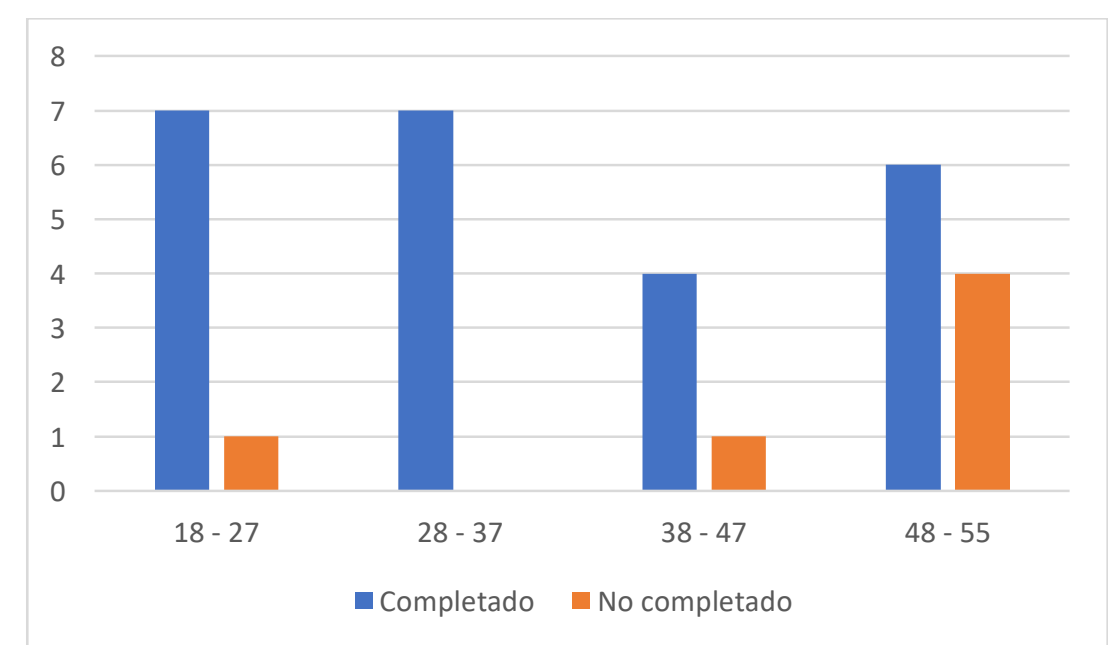

Gráfico 3 Registros con información completa e incompleta en la segunda etapa 


\section{RELEVANCIA DE LOS SISTEMAS PERSONALES DE SALUD DURANTE LA PANDEMIA DE COVID-19 EN MÉXICO}

La valoración del profesional de salud y sus opiniones se presentan en el gráfico 4.

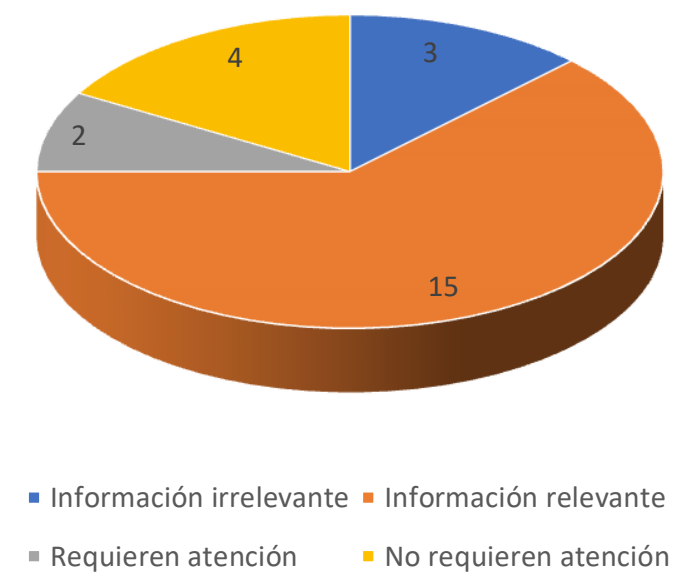

Gráfico 4 Comentarios del profesional de salud en la segunda etapa

Puede observarse un incremento en la exactitud de las valoraciones debido a que contó con una mayor cantidad de información relevante. Adicionalmente, hubo una reducción en el número de expedientes con información irrelevante. En los casos en los que no se pudo establecer una valoración, las razones fueron la falta de información en algunas secciones o un número limitado de eventos reportados. El uso de secciones abiertas para el usuario aumentó la cantidad de información irrelevante o incompleta; por ejemplo: una descripción demasiado general de un malestar. La información más relevante se localizaba en los expedientes enviados por pacientes que habían tenido un encuentro previo con el médico. Un caso importante fue el diagnóstico de un padecimiento de una persona que no había tenido un encuentro previo con el profesional tan solo basado en sus anotaciones.

La herramienta probó ser útil también para valoraciones y seguimiento a personas infectadas con COVID-19. La aplicación ofreció al paciente un espacio para registrar y mantener los seguimientos solicitados por el profesional y enviarle la información en el momento en que le fuera solicitado. Aunque en esta parte del desarrollo no se implementa una comunicación bidireccional, es una posibilidad que ofrecen algunos productos de este tipo. Sin embargo, estos requieren que la información se encuentre centralizada y no distribuida, lo cual puede analizarse como trabajo futuro.

Las ventajas que la aplicación ofreció a los actores se resumen en la tabla 3.

Tabla 3. Ventajas para los actores

\begin{tabular}{|c|c|}
\hline Pacientes & Médicos \\
\hline $\begin{array}{l}\text { El usuario es el dueño de su información } \\
\text { de salud, y es el responsable de }\end{array}$ & $\begin{array}{l}\text { El médico recibe sólo las secciones del } \\
\text { reporte que requiere en el caso del }\end{array}$ \\
\hline
\end{tabular}




\section{RELEVANCIA DE LOS SISTEMAS PERSONALES DE SALUD DURANTE LA PANDEMIA DE COVID-19 EN MÉXICO}

mantenerla y compartirla al profesional que desee.

La información compartida cumple con los estándares y lineamientos aplicables tanto nacionales como internacionales. seguimiento o bien puede recibir toda la información histórica del paciente en caso de requerirla.

Si el sistema es alimentado adecuadamente, el profesional puede tomar en cuenta toda la sintomatología y farmacología involucrada en la historia y no solo confiar en la memoria del paciente, lo que puede traducirse en un diagnóstico más certero.

El sistema permite capturar cada sección de acuerdo a las necesidades del usuario.

El paciente puede capturar en la bitácora de salud los eventos que considere importantes, de los cuales pueden obtenerse nuevos patrones.

El paciente se empodera y se compromete como actor en el mantenimiento y cuidado de su salud.
El médico puede obtener la evolución de cada procedimiento en el tiempo en el que lo requiera.

El médico puede recibir el reporte del PHR previo a la consulta del paciente, lo que agiliza los tiempos de visita.

El médico puede almacenar las diferentes versiones recibidas de los reportes y hacer las veces de sus expedientes clínicos, pues contienen toda la información obligatoria.

Fuente: Elaboración propia.

Es fundamental hacer notorio que el propósito de esta app es el de mejorar la comunicación médico - paciente e involucrar a los pacientes en el cuidado de su salud; y que no se pretende sustituir al médico en su papel de experto. La finalidad de la app es facilitar al profesional la elaboración del diagnóstico de una manera más exacta e informada, ofreciéndole también un documento que puede hacer las veces de expediente clínico. Es innegable la eficiencia de la versión en papel, pero es sabido que tiende a ser difícil de manejar, almacenar y fácilmente olvidable. Incorporar la tecnología demostró involucrar de manera más sencilla a los jóvenes; y la necesidad de considerar las recomendaciones para una mayor usabilidad entre las personas con menores conocimientos tecnológicos.

\section{CONCLUSIONES Y TRABAJO A FUTURO}

En la sociedad mexicana reina la desconfianza hacia compartir información personal por cualquier medio. Esto se pudo comprobar durante la realización de este proyecto pues ambos actores fueron al principio renuentes; los participantes tenían la desconfianza de como iba a ser manejada y almacenada su información de salud. En este sentido, esa desconfianza se minimizó cuando se dieron cuenta que ellos eran los guardianes de su propia información. Por el lado de los médicos, se identificaron dos principales causas de desconfianza hacia el uso de un PHR: la primera era la idea de 


\section{RELEVANCIA DE LOS SISTEMAS PERSONALES DE SALUD DURANTE LA PANDEMIA DE COVID-19 EN MÉXICO}

que la aplicación estuviera diseñada con el fin de hacer diagnósticos médicos. Por otro lado, estaba el hecho de confiar en información recabada por sus propios pacientes y no por ellos.

En este sentido, la situación de pandemia que todavía está presente en el mundo obligó a incluir a la tecnología en todos los aspectos de la vida cotidiana e hizo que los médicos aprendieran a confiar en otras formas para realizar su trabajo. Conforme los actores involucrados en la prestación de servicios de salud se den a la tarea de formar pacientes informados y comprometidos en la prevención y mantenimiento del estado óptimo de la salud, contra el correctivo y de recuperación de la salud; esto conducirá a una mayor robustez del Sistema de Salud Nacional, lo cual se traducirá en un incremento en el nivel económico de las familias y la reducción de las estadísticas en enfermedades crónico-degenerativas, junto con sus costos asociados. El objetivo de establecer a la cultura de la prevención como el comportamiento ideal de todos los mexicanos requiere de esfuerzos conjuntos de los sectores de gobierno, educativos y de salud; dotando a los usuarios de las herramientas adecuadas para esto.

Las anotaciones del usuario son un elemento que queda fuera de los estándares. Esto era una situación esperada pues los estándares están pensados desde el punto de vista del intercambio entre profesionales o prestadores de servicios y, por tanto, el usuario no está considerado como uno de los autores. Para subsanar esta situación se adecuó el schema y se solicitó un OID ante las autoridades correspondientes, sin obtener una respuesta satisfactoria. Por lo que durante esta etapa solo se trabajó con el schema modificado.

La incorporación de herramientas tecnológicas como los wearables para que las mediciones biométricas se agreguen de forma automática a eventos de salud; así como herramientas y cuestionarios estandarizados que permitan detectar otros factores de riesgo como la obesidad y enfermedades cardiacas, así como el involucramiento de instituciones de salud y profesionales de salud son algunos de los trabajos a futuro para esta investigación.

\section{REFERENCIAS}

AHIMA e-HIM Personal Health Record Work Group. (2005). Defining the Personal Health Record. Journal of AHIMA 76(6), 24-25. https://n9.cl/cpqth

Constitución Política de los Estados Unidos Mexicanos, 4 (1917). https://n9.cl/xiclj

Dantés, O. G., Sesma, S., \& Becerril, V. M. (2011). Sistema de salud de México. Salud Pública de México, 53(0). https://n9.cl/of9xp

Dirección General de Información en Salud. (2011). Manual del expediente clínico electrónico. https://n9.cl/4lulo 


\section{RELEVANCIA DE LOS SISTEMAS PERSONALES DE SALUD DURANTE LA PANDEMIA DE COVID-19 EN MÉXICO}

Dirección General de Información en Salud. (2016). Guía para la Elaboración de Resumen Clínico. https://n9.cl/u6myh

HL7 International. (2011). HL7 Standards Product Brief-HL7 Implementation Guide for CDA® R2: Plan-to-Plan Personal Health Record (PHR) Data Transfer, Release 1 / $H L 7$ International. https://n9.cl/1u4x

HL7 International. (2019). About Health Level Seven International. https://n9.cl/ee5k9

HL7 Organization. (2019). Health Level Seven (HL7) [Generic Template]. https://www.siemens-healthineers.com/mx/services/it-standards/hl7

Instituto Mexicano del Seguro Social. (2019, mayo). Acercando el IMSS al Ciudadano. https://n9.cl/qe2b3

Kaelber, D. C., Jha, A. K., Johnston, D., Middleton, B., \& Bates, D. W. (2008). A Research Agenda for Personal Health Records (PHRs). Journal of the American Medical Informatics Association: JAMIA, 15(6), 729-736. https://doi.org/10.1197/jamia.M2547

Leal, H. V., Campos, R. M., Domínguez, C. B., \& Sheissa, R. C. (2011). Un expediente clínico electrónico universal para México: Características, retos y beneficios. 10. https://n9.cl/89fnq

Navarro, M. F. (2020). Mexicanos, con alta vulnerabilidad por pandemia de coronavirus, obesidad y diabetes • Forbes México. Forbes México. https://n9.cl/rzmlk

Realm. (2019). Realm: Create reactive mobile apps in a fraction of the time. https://n9.cl/j50e

Secretaría de Salud. (2012). NORMA Oficial Mexicana NOM-024-SSA3-2012, Sistemas de información de registro electrónico para la salud. Intercambio de información en salud. https://n9.cl/aehku

Secretaría de Salud. (2018). Gobierno de México. Encuentra la unidad médica más cercana con RadarCiSalud. https://n9.cl/ov8jz

\section{CONTRIBUCIONES DE AUTORES, FINANCIACIÓN Y AGRADECIMIENTOS}

Contribuciones de los autores: Conceptualización, Mendoza Olguín Gustavo Emilio y Pérez de Celis Herrero Concepción; metodología, Mendoza Olguín Gustavo Emilio, Pérez de Celis Herrero Concepción, Somodevilla García María Josefa y Mendoza 


\section{RELEVANCIA DE LOS SISTEMAS PERSONALES DE SALUD DURANTE LA PANDEMIA DE COVID-19 EN MÉXICO}

Olguín Iván Agustín; software, Mendoza Olguín Gustavo Emilio; validación, Mendoza Olguín Iván Agustín, Pérez De Celis Herrero Concepción y Somodevilla García María Josefa; análisis formal, Mendoza Olguín Gustavo Emilio, Pérez De Celis Herrero Concepción y Somodevilla García María Josefa; investigación, Mendoza Olguín Gustavo Emilio; recuerdos, Mendoza Olguín Gustavo Emilio; curación de datos, Mendoza Olguín Gustavo Emilio y Mendoza Olguín Iván Agustín; redacción preparación del borrador original, Mendoza Olguín Gustavo Emilio; redacción-revisión y edición, Mendoza Olguín Gustavo Emilio, Pérez de Celis Herrero Concepción, Somodevilla García María Josefa y Mendoza Olguín Iván Agustín; visualización, Mendoza Olguín Gustavo Emilio, Pérez de Celis Herrero Concepción, Somodevilla García María Josefa y Mendoza Olguín Iván Agustín; supervisión, Pérez de Celis Herrero Concepción y Somodevilla García María Josefa administración de proyectos, Mendoza Olguín Gustavo Emilio. Todos los autores han leído y aceptado la versión publicada del manuscrito.

Financiación: Esta investigación fue realizada dentro del programa de Becas de Maestría de CONACYT.

Conflicto de intereses: Ninguno

\section{IIAUTORES}

\section{Mendoza Olguín Gustavo Emilio}

Maestro en Ciencias de la Computación en la Benemérita Universidad Autónoma de Puebla con intereses por la investigación de bases de datos, minería de datos y representación de la información.

Orcid ID: https://orcid.org/0000-0001-7164-4987

\section{Mendoza Olguín Iván Agustín}

Médico especialista en Urgencias por el Instituto Politécnico Nacional y la Benemérita Universidad Autónoma de Puebla. Actualmente trabaja en el Instituto Mexicano del Seguro Social y se ha interesado por el área de informática de la salud.

Orcid ID: https://orcid.org/0000-0002-2208-3148

\section{Pérez de Celis Herrero María de la Concepción}

Docteur 3éme Cycle en Informatique, Université Pierre et Marie Curie (Paris 6), actualmente es Profesor-Investigador Titular B en la Facultad de Ciencias de la Computación de la Benemérita Universidad Autónoma de Puebla, Puebla, México.

Orcid ID: https://orcid.org/0000-0002-2208-3148 


\section{RELEVANCIA DE LOS SISTEMAS PERSONALES DE SALUD DURANTE LA PANDEMIA DE COVID-19 EN MÉXICO}

\section{Somodevilla García María Josefa}

Dra. en Ciencias Computacionales egresada en 2003 de Tulane University, New Orleans, LA, USA. Sus áreas de investigación son Big Data Analytics y Natural Language Processing. Es autora de 60 artículos de investigación y ha dirigido 80 tesis de grado en los niveles de licenciatura, maestría y doctorado.

Orcid ID: https://orcid.org/0000-0002-1972-2252 